\title{
AGN feedback in galaxy groups: a joint GMRT/X-ray study
}

\author{
S. Giacintucci ${ }^{* \dagger}$, J. M. Vrtilek*, E. O'Sullivan*, S. Raychaudhury**, L. P. \\ David $^{*}$, T. Venturi ${ }^{\dagger}$, R. Athreya ${ }^{\ddagger}$ and M. Gitti ${ }^{\S}, *$ \\ ${ }^{*}$ Harvard-Smithsonian Center for Astrophysics, 60 Garden St, Cambridge, MA 02138, USA \\ $\dagger$ INAF-IRA, via Gobetti 101, I-40129, Bologna, Italy \\ ${ }^{* *}$ University of Birmingham, Edgbaston, Birmingham, UK \\ ${ }^{\ddagger} I I S E R$, Pune, Maharashtra 411008 , India India \\ ${ }^{\S}$ Dept. of Astronomy, University of Bologna \& INAF OABo, via Ranzani 1, I-40127, Bologna, Italy
}

\begin{abstract}
We present an ongoing study of 18 nearby galaxy groups, chosen for the availability of Chandra and/or XMM-Newton data and evidence for AGN/hot intragroup gas interaction. We have obtained 235 and $610 \mathrm{MHz}$ observations at the GMRT for all the groups, and 327 and $150 \mathrm{MHz}$ for a few. We discuss two interesting cases - NGC 5044 and AWM 4 - which exhibit different kinds of AGN/hot gas interaction. With the help of these examples we show how joining low-frequency radio data (to track the history of AGN outbursts through emission from aged electron populations) with X-ray data (to determine the state of hot gas, its disturbances, heating and cooling) can provide a unique insight into the nature of the feedback mechanism in galaxy groups.
\end{abstract}

Keywords: Galaxy groups, Radio galaxies, X-ray observations

PACS: 98.54.Gr,98.65.-r,98.65.Bv

\section{INTRODUCTION}

Several feedback mechanisms have been invoked to balance the cooling process in the hot X-ray emitting gas halo of clusters and groups of galaxies. These include gravitational heating, supernovae, subcluster mergers, thermal conduction, and AGNdriven outbursts. In rich clusters, the radio outbursts from the AGN harboured in the central galaxy appear to be the dominant source of heating. However most of the galaxies and baryonic matter in the Universe reside in less massive units, such as poor clusters and groups of galaxies. Thus the study of feedback in these environments is fundamental to understand the mechamism by which feedback operates and how it has influenced the formation and thermal history of most of the baryons in the Universe.

\section{OUR ONGOING GMRT/X-RAY STUDY OF GALAXY GROUPS}

To investigate the impact of AGN feedback in galaxy groups, we are carrying out an in-depth study of a representative collection of 18 galaxy groups (listed in Tab. 1), using low (and multi) frequency radio observations, obtained at the Giant Metrewave Radio Telescope (GMRT), and deep archival Chandra and XMM-Newton X-ray data. The groups were chosen on the basis of structures, either in the X-ray surface brightness and temperature maps or radio morphology, which strongly indicate interaction between 
TABLE 1. List of galaxy groups and status of the GMRT observations.

\begin{tabular}{|c|c|c|c|c|c|}
\hline Group name & $235 \mathrm{MHz}$ & $610 \mathrm{MHz}$ & Group name & $235 \mathrm{MHz}$ & $610 \mathrm{MHz}$ \\
\hline UGC 408* & Aug 2007 & Aug 2008 & NGC 3411 & Aug 2006 & Feb 2008 \\
\hline NGC 315 & Feb 2008 & Aug 2008 & NGC 4636 & Aug 2006 & Feb 2008 \\
\hline NGC 383 & Feb 2008 & Aug 2008 & HCG 62 & Feb 2008 & Feb 2008 \\
\hline NGC 507 & July 2006 & Aug 2008 & NGC $5044^{* \dagger}$ & Feb 2008 & Feb 2008 \\
\hline NGC 741 & Aug 2006 & Aug 2007 & NGC 5813* & - & Aug 2008 \\
\hline HCG 15 & Aug 2006 & Aug 2008 & NGC 5846 & Aug 2006 & - \\
\hline NGC 1407 & July 2006 & Aug 2008 & $\mathrm{AWM} 4^{* *}$ & Aug 2006 & Jul 2006 \\
\hline NGC 1587 & Aug 2006 & Aug 2008 & NGC 6269 & Feb 2008 & Feb 2008 \\
\hline MKW $02 \ddagger$ & Aug 2003 & July 2005 & NGC $7626^{*}$ & Aug 2007 & Aug 2008 \\
\hline
\end{tabular}

the radio source and the intragroup medium. All groups, except two, were observed with the GMRT both at $610 \mathrm{MHz}$ and $235 \mathrm{MHz}$ during Cycle 12, 14 and 15. The observing period for each frequency is given in Tab. 1. GMRT observations at $327 \mathrm{MHz}$ and 150 $\mathrm{MHz}$ were also obtained for few targets (see notes to Tab. 1) as part of the followup ongoing at these frequencies. The data from all observations in Tab. 1 have been completely reduced. The sensitivity achieved in the final images is in the range 35-100 $\mu \mathrm{Jy}$ at $610 \mathrm{MHz}$ and $0.2-1 \mathrm{mJy}$ at $235 \mathrm{MHz}$ for a typical observing time of $\sim 2-3$ hours on source. The ongoing analysis shows that the sources are diverse, covering a range of spatial scales and total powers, from classic double FR-I to core-halo radio sources (Giacintucci et al. in preparation).

\section{A CLOSER VIEW OF TWO REMARKABLE GALAXY GROUPS}

We have selected two systems from the above sample - NGC 5044 and AWM 4 - which exhibit different kinds of AGN/hot gas interaction, to show how the combination of deep $\mathrm{X}$-ray data and high-sensitivity low frequency radio observations can provide a unique insight into the nature of the feedback mechanism in galaxy groups.

NGC 5044. The new Chandra observation reveals that the group hosts many small radio quiet cavities, filaments, and a semi-circular cold front (Fig. 1; David et al. 2009). The GMRT $610 \mathrm{MHz}$ image (right) shows the radio core and a lobe which extends along a filament of cold gas. The $235 \mathrm{MHz}$ emission (left) is much more extended, with little overlap with the $610 \mathrm{MHz}$ image. The emission fills the S cavity, curves toward the W just behind the cold front, and then sharply bends by $\sim 90^{\circ}$. A second component, a detached radio lobe, is visible toward the S-E, and its western edge is coincident with the cold front, suggesting that the relativistic material in this structure might have been produced by an earlier outburst and that it is currently compressed by the motion of NGC 5044 toward the S-E. The lack of any detected emission at $610 \mathrm{MHz}$ in the same 

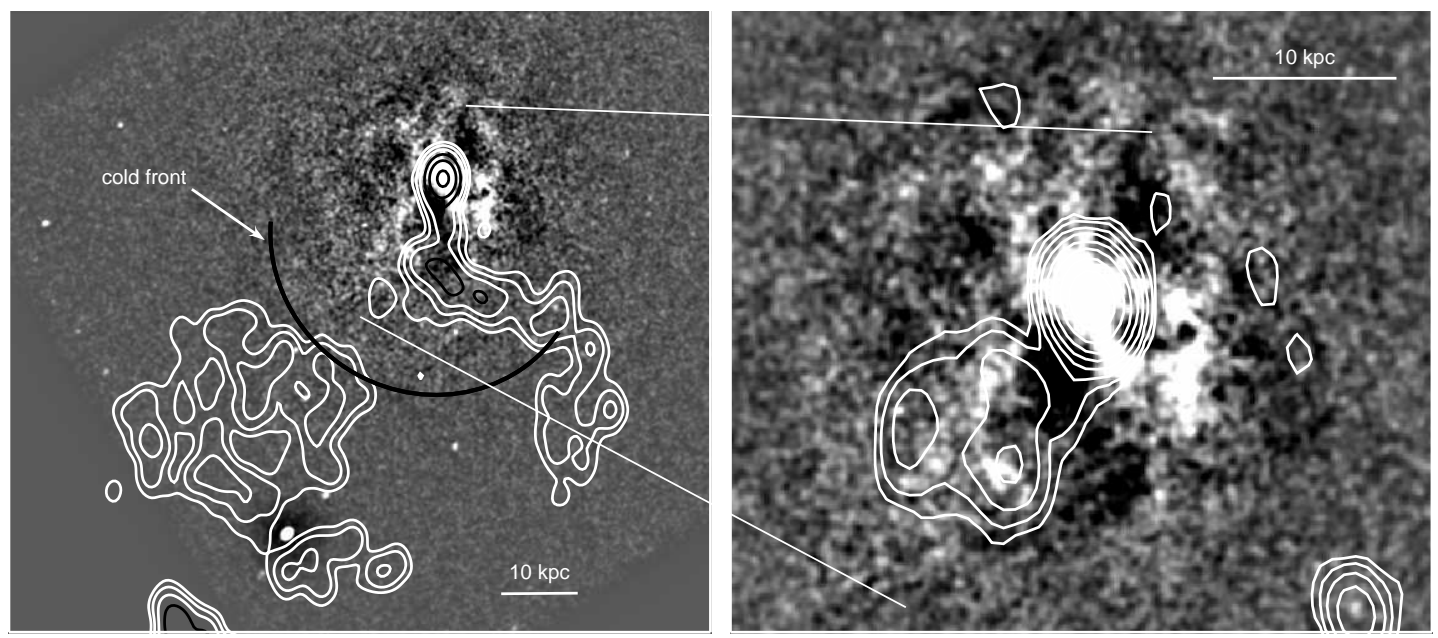

FIGURE 1. NGC 5044: GMRT $235 \mathrm{MHz}$ (left) and $610 \mathrm{MHz}$ contours (right) on the Chandra unsharp masked image in the 0.3-2.0 keV band (David et al. 2009). The radio beam is $22^{\prime \prime} \times 16^{\prime \prime}$ and $18^{\prime \prime} \times 16^{\prime \prime}$, respectively. The lowest contour is shown at $3 \sigma=0.75 \mathrm{mJy} \mathrm{b}^{-1}$ and $0.075 \mathrm{mJy} \mathrm{b}^{-1}$, respectively.
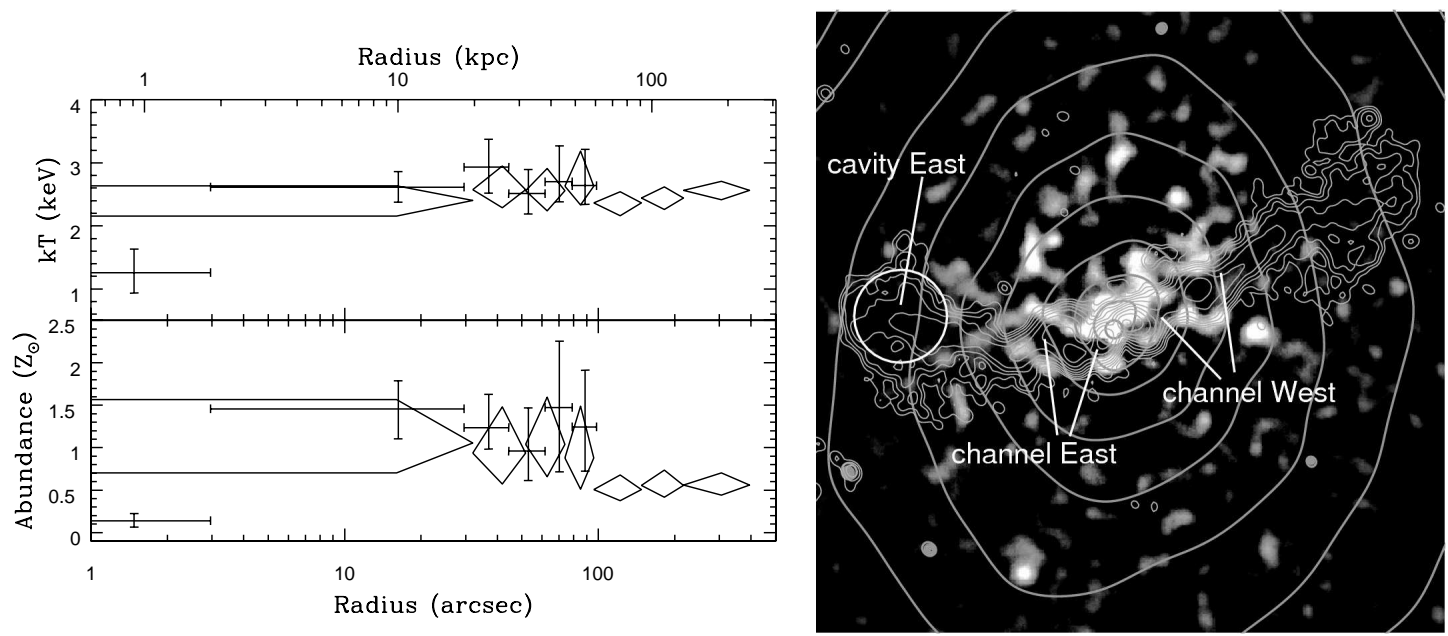

FIGURE 2. AWM 4. Left: Chandra radial profiles of deprojected temperature and abundance. Crosses and diamonds refer to fits carried out in the $0.7-7.0 \mathrm{keV}$ band with 8000 net counts/spectrum $(370 \mathrm{cts}$ for the central bin) and 12000 net counts/spectrum, respectively. Right: GMRT $610 \mathrm{MHz}$ contours (beam $5^{\prime \prime} \times 4^{\prime \prime}$; lowest contour at $3 \sigma=0.15 \mathrm{mJy} \mathrm{b}^{-1}$ ), superposed on the Chandra residual image, after subtraction of the best-fitting surface brightness model.

region as the $235 \mathrm{MHz}$ emission indicates that the radio spectrum must be very steep. The GMRT data thus appear to reveal 2 (or possibly 3) separate outbursts. The youngest outburst can be identified with the $610 \mathrm{MHz}$ emission, and the oldest burst with the detached radio lobe.

AWM 4. Previous XMM-Newton observations showed AWM 4 to be isothermal at $\sim 2.5 \mathrm{keV}$ out to at least $160 \mathrm{kpc}$ from the centre, even though the cooling time in 
the middle is $\sim 2$ Gyr (O'Sullivan et al. 2005). Its powerful central radio galaxy was proposed as the most likely source of heating. Our deep GMRT observations at 235, 327 and $610 \mathrm{MHz}$, presented in Giacintucci et al. (2008), revealed the full extent of the radio source and allowed us to determine its age, orientation, energy and physical parameters. However, with no indications of X-ray cavities associated with the radio source, the question of the coupling between jets and intra-group gas remained unresolved (see also Gastaldello et al. 2008). Our new $80 \mathrm{ksec}$ Chandra image reveals the small-scale galactic corona surrounding the AGN; the radial temperature and abundance profiles show a clear decrease in the central $\sim 2 \mathrm{kpc}$-radius region (Fig. 2; left), corresponding to the corona. The existence of this corona might exlain the long timescale of the outburst, as it fuels the AGN but is largely unaffected by the radio jets. We do not detect clear cavities in the Chandra image associated with the radio lobes. Some weak X-ray features are visible in the residual image obtained after subtraction of the best-fitting surface brightness model, shown in Fig. 2 (right) with overlaid the GMRT $610 \mathrm{MHz}$ contours. A faint depression is visible in the region of the eastern lobe, and two channel-like structures appear to be associated with the inner jets. No cavity is detected in the western lobe. The detailed analysis of these features and their implications is ongoing (O'Sullivan et al. in prep.).

\section{SUMMARY}

Our ongoing X-ray/GMRT study of the AGN feedback in galaxy groups shows that low frequency observations play a crucial role in the estimate of the total radio energy input from the central AGN and are important in the study of the history of the energy injection and transfer, since they can reveal old, steep-spectrum radio emission and structures related to previous AGN outbursts (e.g., NGC 5044). As shown by our analysis of AWM 4, the combination of the low frequency information with deep and high resolution X-ray data offers a unique tool to investigate the AGN/hot gas interaction (see also Gitti et al., these proceedings). This is of fundamental importance to determine the thermal history of the intragroup and intracluster medium and shed light on the role of the AGN feedback on the formation and evolution of galaxies in these environments.

\section{ACKNOWLEDGMENTS}

We thank the staff of the GMRT for their help during the observations. GMRT is run by the National Centre for Radio Astrophysics of the Tata Institute of Fundamental Research. We thank C. Jones, W. Forman, P. Mazzotta, M. Murgia, and T. Ponman.

\section{REFERENCES}

1. L. P. David, C. Jones, S. Giacintucci, et al., AJ, 2009, in press, arXiv:0905.0654

2. F. Gastaldello, D. A. Buote, F. Brighenti, W. G. Mathews, 2008, ApJ, 673, L17

3. S. Giacintucci, T. Venturi, M. Murgia, et al., 2007, A\&A, 476, 99

4. S. Giacintucci, J. M. Vrtilek, M. Murgia, et al., 2008, ApJ, 682, 186

5. M. Gitti, E. O'Sullivan, S. Giacintucci, et al., these proceedings

6. E. O'Sullivan, J. M. Vrtilek, J. C Kempner, et al., 2005, MNRAS, 357, 1134 